F\&M96/3

hep-ph/9610541

\title{
PRODUCTION OF A HIGGS BOSON PLUS TWO JETS IN HADRONIC COLLISIONS
}

\author{
Russel P. Kauffman, Satish V. Desai and Dipesh Risal \\ Department of Physics and Astronomy \\ Franklin and Marshall College, Lancaster, PA 17604
}

\begin{abstract}
We consider the production of a Standard Model Higgs boson accompanied by two jets in hadronic collisions. We work in the limit that the top quark is much heavier than the Higgs boson and use an effective Lagrangian for the interactions of gluons with the Higgs boson. In addition to the previously computed four-gluon process, we compute the the amplitudes involving two quarks, two gluons and the Higgs boson and those involving four quarks and the Higgs boson. We exhibit the form of our results in the small- $p_{\mathrm{H}}$ and factorization limits. We present numerical results for $\sqrt{S}=14 \mathrm{TeV}$ and $\sqrt{S}=2 \mathrm{TeV}$. We find that the dominant processes are $g g \rightarrow g g H$ and $q g \rightarrow q g H$ with the former (latter) contributing about $60 \%(40 \%)$ of the cross section at $\sqrt{S}=14 \mathrm{TeV}$ and the two processes each contributing about half the cross section at $\sqrt{S}=2 \mathrm{TeV}$. All other processes are negligible at both energies.
\end{abstract}

September 1996 


\section{Introduction}

The Higgs boson is the last remaining undiscovered element of the Standard Model. Discovery of a Higgs boson (or more than one) would confirm that the Higgs mechanism is the source of electro-weak symmetry breaking while convincing evidence that Higgs bosons do not exist would necessitate another explanation for electro-weak symmetry breaking. Thus, the search for the Higgs boson is one of the fundamental quests of modern high energy physics. Current published experimental results set a lower limit on the Higgs boson mass of about $60 \mathrm{GeV}$ [1] while the $e^{+} e^{-}$collider LEPII can be expected to extend this limit to somewhere near $80 \mathrm{GeV}$.

In this paper, we are concerned with the production of Higgs bosons in hadronic collisions. We are particularly interested in the so-called "intermediate mass" Higgs boson, i.e., one in the mass region $80 \mathrm{GeV} \leq M_{\mathrm{H}} \leq 200 \mathrm{GeV}$, although, as we will argue, our results have a somewhat larger range of applicability. Experimentally this is an extremely difficult region in which to see the Higgs boson, due to the large backgrounds to the common Higgs boson decay channels. Hence it is vital to have precise predictions for the production cross section as well as for the distribution of the produced Higgs bosons in transverse momentum and rapidity. The probability of extra particles, be they jets, W's or top quarks, being produced along with the Higgs boson, also impacts its detection. Accompanying particles may act as tags or be confused with the Higgs decay products.

Here we discuss the production of the Higgs boson accompanied by two jets. The cross section for $g g \rightarrow g g H$ was calculated previously [2]. We compute the contributions needed for the total Higgs boson plus two jet cross section: $g g \rightarrow q q H, q g \rightarrow q g H, q q \rightarrow g g H, q q \rightarrow q q H$, where ' $q$ ' stands generically for a quark or anti-quark of undetermined flavor. We consider only the QCD generated processes, that is, extra quark or gluons lines attached to the basic gluon-gluon-Higgs-boson interaction. (The electroweak process, $q q \rightarrow q q H$ through $W W$ or $Z Z$ fusion does not interfere with the all quark process considered here. The interference term would be proportional to the trace of a single $S U(3)$ generator and so vanishes.)

We will work in the limit in which the top quark is much heavier than the Higgs boson and all of the energy scales in the problem. Since experiments

[3] place $m_{\text {top }} \simeq 175 \mathrm{GeV}$, this limit is relevant for the intermediate mass Higgs boson. This limit is also relevant for consideration of soft and collinear 
radiation surrounding the production of the Higgs boson.

The organization of the paper is as follows. The effective Lagrangian is discussed in Section 2. Section 3 contains the spinor product formalism in which the amplitudes will be computed. The amplitudes for a Higgs boson plus two or three massless particles are computed in Section 4. For completeness the Higgs boson plus four gluon amplitude is presented in Section 5. Sections 6 and 7 contain the calculations of the amplitude involving a Higgs boson plus a quark anti-quark pair and two gluons and the amplitude for a Higgs boson plus two quark anti-quark pairs, respectively. The limit of our results when the momentum of the Higgs is small is presented in Section 8 and their behaviour in the factorization limits is presented in Section 9. Section 10 contains our numerical results and the Appendix contains the squares of the various amplitudes.

\section{The Effective Lagrangian}

The production mechanism in which we are interested is $g g \rightarrow H$ which occurs through a quark loop where the only numerically important contribution is that of the top quark. In the limit in which the top quark is heavy, $m_{\text {top }} \gg M_{\mathrm{H}}$, the cross section can be computed via the following effective Lagrangian [1]

$$
\mathcal{L}_{\text {eff }}=-\frac{1}{4} A H G_{\mu \nu}^{A} G^{A \mu \nu},
$$

where $G_{\mu \nu}^{A}$ is the field strength of the $\mathrm{SU}(3)$ color gluon field and $H$ is the Higgs-boson field. The effective coupling $A$ is given by $A=\alpha_{s} /(3 \pi v)$, where $v$ is the vacuum expectation value parameter, $v^{2}=\left(G_{F} \sqrt{2}\right)^{-1}=(246 \mathrm{GeV})^{2}$. The effective Lagrangian generates vertices involving the Higgs boson and two, three or four gluons. The associated Feynman rules are displayed in Fig 1. The two-gluon-Higgs-boson vertex is proportional to the tensor

$$
H^{\mu \nu}\left(p_{1}, p_{2}\right)=g^{\mu \nu} p_{1} \cdot p_{2}-p_{1}^{\nu} p_{2}^{\mu} .
$$

The vertices involving three and four gluons and the Higgs boson are proportional to their counterparts from pure QCD:

$$
V^{\mu \nu \rho}\left(p_{1}, p_{2}, p_{3}\right)=\left(p_{1}-p_{2}\right)^{\rho} g^{\mu \nu}+\left(p_{2}-p_{3}\right)^{\mu} g^{\nu \rho}+\left(p_{3}-p_{1}\right)^{\nu} g^{\rho \mu}
$$


and

$$
\begin{aligned}
X_{a b c d}^{\mu \nu \rho \sigma} & =f_{\text {abe }} f_{c d e}\left(g^{\mu \rho} g^{\nu \sigma}-g^{\mu \sigma} g^{\nu \rho}\right)+f_{\text {ace }} f_{b d e}\left(g^{\mu \nu} g^{\rho \sigma}-g^{\mu \sigma} g^{\nu \rho}\right) \\
& +f_{\text {ade }} f_{b c e}\left(g^{\mu \nu} g^{\rho \sigma}-g^{\mu \rho} g^{\nu \sigma}\right) .
\end{aligned}
$$

It is straightforward to use this Lagrangian to obtain the $\mathcal{O}\left(\alpha_{s}^{3}\right)$ contributions to the process $g g \rightarrow H$ [5, 6]. These radiative corrections increase the lowest order rate by a factor of 1.5 to 2 . As a by-product of the calculation of the $\mathcal{O}\left(\alpha_{s}^{3}\right)$ radiative corrections to $g g \rightarrow H$, one also obtains the cross section for $g g \rightarrow H g$.

If the Higgs boson mass is of the same order as the top quark mass or larger the approximation entailed in the effective Lagrangian breaks down. However, even if $M_{\mathrm{H}}$ is not much smaller than $m_{t}$, the results of the effective Lagrangian can be applied, after some modification, in the the soft and/or collinear regime. Factorization requires that as a gluon becomes soft or two particles become collinear an amplitude must factor into a divergent piece and a non-divergent piece, the divergent piece being independent of the hard process. Applied to the case of the Higgs-jet-jet amplitudes, when both outgoing jets have small $p_{\mathrm{T}}$ (compared to the lowest scale in the problem, $M_{\mathrm{H}}$ or $m_{t}$ ) factorization requires that the dependence on $M_{\mathrm{H}} / m_{t}$ must be the same as the lowest order $H g g$ amplitude. This was shown explicitly in Ref. [7] for the processes $g g \rightarrow g H, q g \rightarrow q H$ and $q \bar{q} \rightarrow g H$. Since in this limit the only dependence on $m_{t}$ is in the overall factor, the result derived from the effective Lagrangian may simply be rescaled in order to be applied to the case when $m_{t}$ is not much larger than $M_{\mathrm{H}}$.

\section{Spinor Product Formalism}

We are interested in processes in which all the particles except the Higgs boson are massless. Each amplitude can be expressed in terms of spinors in a Weyl basis. For light-like momentum $p$ and helicity $\lambda= \pm 1$ we introduce spinors [8, 9]

$$
\begin{aligned}
|p \pm\rangle & =\frac{1}{2}\left(1 \pm \gamma_{5}\right) u(p)=\frac{1}{2}\left(1 \mp \gamma_{5}\right) v(p) \\
\langle p \pm|=\bar{u}(p) \frac{1}{2}\left(1 \mp \gamma_{5}\right) & =\bar{v}(p) \frac{1}{2}\left(1 \pm \gamma_{5}\right) .
\end{aligned}
$$


Polarization vectors for massless vector bosons can be written in terms of these spinors. For a gluon of momentum $k$ and positive or negative helicity

$$
\epsilon_{ \pm}^{\mu}=\frac{\left\langle q \pm\left|\gamma^{\mu}\right| k \pm\right\rangle}{\sqrt{2}\langle q \mp \mid k \pm\rangle}
$$

where the reference momentum $q$ satisfies $q^{2}=0$ and $q \cdot k \neq 0$ but is otherwise arbitrary; the freedom of the choice of $q$ is a reflection of gauge invariance. Each helicity amplitude can be expressed in terms of products of these spinors:

$$
\begin{aligned}
& \langle p-\mid q+\rangle=-\langle q-\mid p+\rangle \equiv\langle p q\rangle, \\
& \langle p+\mid q-\rangle=-\langle q+\mid p-\rangle \equiv[p q], \\
& \langle p-\mid q-\rangle=\langle q+\mid p+\rangle=\langle p p\rangle=[p p]=0 .
\end{aligned}
$$

The spinor product $\langle p q\rangle$ and $[p q]$ are complex square roots of $2 p \cdot q$,

$$
\begin{aligned}
& \langle p q\rangle[q p]=2 p \cdot q, \\
& \langle p q\rangle^{*}=\operatorname{sign}(p \cdot q)[q p] .
\end{aligned}
$$

Using the identities

$$
\not p=|p+\rangle\langle p+|+| p-\rangle\langle p-|
$$

and

$$
\left\langle p \pm\left|\gamma^{\mu}\right| q \pm\right\rangle \gamma_{\mu}=2(|q \pm\rangle\langle p \pm|+| p \mp\rangle\langle q \mp|,
$$

each amplitude can be written solely in terms of spinor products. The following identity and its complex conjugate are useful for simplifying the results:

$$
\langle p q\rangle\langle r s\rangle=\langle p s\rangle\langle r q\rangle+\langle p r\rangle\langle q s\rangle .
$$

For the remainder of the paper we will use the convention that all the particles are outgoing. The amplitudes for the various processes involving two incoming massless particles and two outgoing massless particles plus a Higgs boson can then be obtained by crossing symmetry. The momenta of the massless particles are labelled $p_{1}, p_{2}, p_{3}, p_{4}$ with the Higgs boson momentum being $p_{\mathrm{H}}$. Our convention is then $p_{1}+p_{2}+p_{3}+p_{4}+p_{\mathrm{H}}=0$. We will use the shorthand notations $\left\langle p_{i} p_{j}\right\rangle=\langle i j\rangle,\left[p_{i} p_{j}\right]=[i j],\left(p_{i}+p_{j}\right)^{2}=S_{i j}$, and $\left(p_{i}+p_{j}+p_{k}\right)^{2}=S_{i j k}$. 


\section{Two and Three Particle Plus Higgs Boson Processes}

As a preliminary to calculating the Higgs plus four-parton amplitudes, we present here the two and three parton amplitudes as examples. These amplitudes also provide the limiting forms of the four-parton amplitudes when two partons become collinear or a gluon becomes soft. The lowest order process by which Higgs bosons are created is $g g \rightarrow H$. The Feynman diagram is given in Fig. 1a. The non-zero helicity amplitudes are ++ and -- . We find

$$
\mathcal{M}^{++}=\frac{1}{2} i A[12]^{2} \delta^{a b}
$$

The amplitude for the - - helicity combination can be obtained by exchanging square brackets for triangle brackets in this expression.

The lowest order processes which produce Higgs bosons with non-zero transverse momentum are $g g \rightarrow g H, q g \rightarrow q H$ and $q \bar{q} \rightarrow g H$. The third process is a crossing of the second. The relevant Feynmam diagrams are shown in Fig.'s 2 and 3. For $g g g H$ the independent helicity amplitudes are (labelled according to helicities of gluons 1,2 and 3 in order)

$$
\begin{aligned}
\mathcal{M}^{+++} & =\frac{g A f_{a b c} M_{\mathrm{H}}^{4}}{\sqrt{2}\langle 12\rangle\langle 23\rangle\langle 31\rangle} \\
\mathcal{M}^{-++} & =\frac{g A f_{a b c}[23]^{3}}{\sqrt{2}[12][13]}
\end{aligned}
$$

The parity conjugate amplitudes can be obtained by exchanging square brackets for triangle brackets and multiplying by -1. Squaring these amplitudes and summing over helicities and colors leads to the known result [10, 5]

$$
\sum|\mathcal{M}(H \rightarrow g g g)|^{2}=\frac{g^{2} A^{2} N\left(N^{2}-1\right)}{S_{12} S_{13} S_{23}}\left(S_{12}^{4}+S_{13}^{4}+S_{23}^{4}+M_{\mathrm{H}}^{8}\right),
$$

where $N=3$ is the number of colors.

The helicity amplitudes for the process $H \rightarrow q \bar{q} g$ can be obtained similarly. Since QCD is helicity conserving in the massless limit, the quark and anti-quark must have opposite helicities. Using the momentum and color 
assignments in Fig. 3 we have (labeling the amplitudes by the helicity of the quark, antiquark and gluon, in that order)

$$
\mathcal{M}^{+-+}=-\frac{i g T_{i j}^{a} A}{\sqrt{2}} \frac{[13]^{2}}{[12]},
$$

where the $S U(3)$ generators are normalized such that $\operatorname{Tr}\left(T^{a} T^{b}\right)=\frac{1}{2} \delta_{a b}$. To get the parity conjugate amplitude $\mathcal{M}^{-+-}$exchange square brackets with triangle brackets. To get the charge conjugates of these two amplitudes, $\mathcal{M}^{-++}$and $\mathcal{M}^{+--}$, respectively, exchange $p_{1} \leftrightarrow p_{2}$. Again, when squared and summed over colors and helicities, these results agree with the known expression [10, 5].

\section{The Higgs Boson Plus Four Gluon Ampli- tude}

The Hgggg amplitude [2] is obtained by summing the 26 Feynamn diagrams detailed in Fig. 4. Unlike the case of Hggg not all the diagrams have the same color structure. To facilitate the cancellations that simplify the amplitude we introduce the dual color decomposition. The scattering amplitude for a Higgs boson and $n$ gluons with external momenta $p_{1}, \ldots p_{n}$, colors $a_{1}, \ldots a_{n}$, and helicities $\lambda_{1}, \ldots \lambda_{n}$ is written as [11, 12, 13]

$$
\mathcal{M}=2 A g^{n-2} \sum_{\text {perms }} \operatorname{tr}\left(T^{a_{1}} \ldots T^{a_{n}}\right) m\left(p_{1}, \epsilon_{1} ; \ldots ; p_{n}, \epsilon_{n}\right),
$$

where the sum is over the non-cyclic permutions of the momenta. This form of the amplitude emerges when the identities

$$
\begin{aligned}
f_{a b c} & =-2 i \operatorname{Tr}\left(T^{a} T^{b} T^{c}-T^{c} T^{b} T^{a}\right), \\
f_{a b e} f_{c d e} & =-2 \operatorname{Tr}\left(\left[T^{a}, T^{b}\right]\left[T^{c}, T^{d}\right]\right)
\end{aligned}
$$

are used to replace the $f_{a b c}$ 's with traces of combinations of $T^{a}$ 's. The utility of the dual decomposition, Eq. 17, comes from the properties of the ordered sub-amplitudes $m\left(p_{1}, \epsilon_{1} ; \ldots ; p_{n}, \epsilon_{n}\right)$, which we abbreviate $m(1, \ldots, n)$ :

1) they are invariant under cyclic permutations of the momenta;

2) they are independently gauge invariant; 
3) $m(1, \ldots, n)=(-1)^{n} m(n, \ldots, 1)$;

4) they satisfy the "dual Ward identity," which for $n=4$ is

$$
m(1,2,3,4)+m(2,1,3,4)+m(2,3,1,4)=0 ;
$$

5) they factorize in the soft gluon limit and in the limit in which two of the gluons are collinear;

6) they are incoherent (to leading order in the number of colors, in general, and completely for $n=4$ ); for $n=4$ one finds

$$
\sum_{\text {colors }}|\mathcal{M}|^{2}=\frac{g^{2} A^{2}}{4} N^{2}\left(N^{2}-1\right) \sum_{\text {perms }}|m(1,2,3,4)|^{2} .
$$

(See Appendix A for the proof.)

The complete set of sub-amplitudes can be obtained from the following three [2]:

$$
\begin{aligned}
& m\left(1^{+}, 2^{+}, 3^{+}, 4^{+}\right)=\frac{M_{\mathrm{H}}^{4}}{\langle 12\rangle\langle 23\rangle\langle 34\rangle\langle 41\rangle} \\
& m\left(1^{-}, 2^{+}, 3^{+}, 4^{+}\right)=-\frac{\left\langle 1-\left|p_{\mathrm{H}}\right| 3-\right\rangle^{2}[24]^{2}}{S_{124} S_{12} S_{14}}-\frac{\left\langle 1-\left|p_{\mathrm{H}}\right| 4-\right\rangle^{2}[23]^{2}}{S_{123} S_{12} S_{23}} \\
& \quad-\frac{\left\langle 1-\left|p_{\mathrm{H}}\right| 2-\right\rangle^{2}[34]^{2}}{S_{134} S_{14} S_{34}}+\frac{[24]}{[12]\langle 23\rangle\langle 34\rangle[41]}\left\{S_{23} \frac{\left\langle 1-\left|p_{\mathrm{H}}\right| 2-\right\rangle}{\langle 41\rangle}\right. \\
& \left.+S_{34} \frac{\left\langle 1-\left|p_{\mathrm{H}}\right| 4-\right\rangle}{\langle 12\rangle}-[24] S_{234}\right\} \\
& m\left(1^{-}, 2^{-}, 3^{+}, 4^{+}\right)=-\frac{\langle 12\rangle^{4}}{\langle 12\rangle\langle 23\rangle\langle 34\rangle\langle 41\rangle}-\frac{[34]^{4}}{[12][23][34][41]} .
\end{aligned}
$$

The structures containing $p_{\mathrm{H}}$ can be expanded in terms of spinor products using Eq. 9 and momentum conservation. For example $\left\langle 1-\left|p_{\mathrm{H}}\right| 3-\right\rangle=$ $-(\langle 12\rangle[23]+\langle 14\rangle[43])$. Permutations of $m\left(1^{+}, 2^{+}, 3^{+}, 4^{+}\right)$are obtained by permuting the momenta in the right side of Eq. 21 identically. Permutations of $m\left(1^{-}, 2^{+}, 3^{+}, 4^{+}\right)$are obtained by permuting $p_{2}, p_{3}$ and $p_{4}$ in the right side of Eq. 22 then using the cyclic and reversal properties of the sub-amplitudes. Permutations of $m\left(1^{-}, 2^{-}, 3^{+}, 4^{+}\right)$are obtained by permuting the momenta in the denominators of the right side of Eq. 23 only. It is straightforward to check that the sub-amplitudes obtained from Eqs. $21-23$ in this way obey the requisite relations. The amplitudes for the other helicity combinations can be obtained (modulo phases) by parity transformations. 


\section{The Higgs Boson Plus Quark Anti-quark and Two Gluon Amplitude}

The $H q \bar{q} g g$ amplitude can be obtained from the Feynman diagrams of Fig. 5. As was the case for the Hgggg amplitude, the calculation can be simplified by judicious choice of color decomposition [14, 12]. The amplitude for a Higgs boson, a quark-anti-quark pair with color indices $i, j$ and $n$ gluons with color indices $a_{1}, \ldots, a_{n}$ can be written:

$$
\mathcal{M}=-i g^{n} A \sum_{\text {perms }}\left(T^{a_{1}} T^{a_{2}} \ldots T^{a_{n}}\right)_{i j} m\left(p 1, \epsilon_{1} ; \ldots ; p_{n}, \epsilon_{n}\right),
$$

where the sum runs over all $n$ ! permutations of the gluons and the subamplitudes $m\left(p 1, \epsilon_{1} ; \ldots ; p_{n}, \epsilon_{n}\right)$ have an implicit dependence on the momenta and helicities of the quark and anti-quark. For the case we are interested in there are only two subamplitudes which we will label as $m(3,4)$ and $m(4,3)$ since the gluon momenta are $p_{3}$ and $p_{4}$. Like the subamplitudes for the pure gluon case these subamplitudes are separately gauge independent and factorize in the soft gluon and collinear particle limits.

As in the case of the $H q \bar{q} g$ amplitude the quark and anti-quark must have opposite helicities. Labelling the helicity amplitudes by the helicity of the quark, anti-quark and the two gluons (in that order) we find

$$
\begin{aligned}
m^{+-++}(3,4) & =\frac{\left\langle 2-\left|p_{\mathrm{H}}\right| 3-\right\rangle^{2}}{S_{124}} \frac{[14]}{\langle 24\rangle}\left(\frac{1}{S_{12}}+\frac{1}{S_{14}}\right)-\frac{\left\langle 2-\left|p_{\mathrm{H}}\right| 4-\right\rangle^{2}}{S_{123} S_{12}} \frac{[13]}{\langle 23\rangle} \\
& +\frac{\left\langle 2-\left|p_{\mathrm{H}}\right| 1-\right\rangle^{2}}{[12]\langle 23\rangle\langle 24\rangle\langle 34\rangle}
\end{aligned}
$$

To get the subamplitude with the other ordering, $m^{+-++}(4,3)$, exchange $p_{3} \leftrightarrow p_{4}$ in this expression. The other independent subamplitudes are

$$
\begin{aligned}
& m^{+-+-}(3,4)=-\frac{\langle 24\rangle^{3}}{\langle 12\rangle\langle 23\rangle\langle 34\rangle}+\frac{[13]^{3}}{[12][14][34]} \\
& m^{+-+-}(4,3)=-\frac{[13]^{2}[23]}{[12][24][34]}+\frac{\langle 14\rangle\langle 24\rangle^{2}}{\langle 12\rangle\langle 13\rangle\langle 34\rangle}
\end{aligned}
$$

The other helicity amplitudes (up to phases) can be obtained by parity $(P)$, Bose symmetry $(B)$ and charge conjugation $(C)$ transformations:

$$
\left|\mathcal{M}^{-+--}\right|^{2} \stackrel{P}{=}\left|\mathcal{M}^{+-++}\right|^{2}, \quad\left|\mathcal{M}^{-+++}\right|^{2} \stackrel{C}{=}\left|\mathcal{M}^{+-++}\right|_{1 \leftrightarrow 2}^{2},
$$




$$
\begin{aligned}
& \left|\mathcal{M}^{+---}\right|^{2} \stackrel{C P}{=}\left|\mathcal{M}^{+-++}\right|_{1 \leftrightarrow 2}^{2}, \quad\left|\mathcal{M}^{-+-+}\right|^{2} \stackrel{P}{=}\left|\mathcal{M}^{+-+-}\right|^{2}, \\
& \left|\mathcal{M}^{+--+}\right|^{2} \stackrel{B}{=}\left|\mathcal{M}^{+-+-}\right|_{3 \leftrightarrow 4}^{2}, \quad\left|\mathcal{M}^{-++-}\right|^{2} \stackrel{B P}{=}\left|\mathcal{M}^{+-+-}\right|_{3 \leftrightarrow 4}^{2} .
\end{aligned}
$$

\section{The Higgs Boson Plus Two Quark Anti- Quark Pair Amplitude}

The remaining processes producing a Higgs boson plus two jets are those involving a combination of four quarks and anti-quarks. In the case where the two pairs are of different flavors the amplitude can be obtained from the Feynman diagram in Fig. 6. In the case when the two pairs are identical there is an additional diagram which can be obtained by switching the $2 \leftrightarrow 4$ in the diagram of Fig. 6. We present the amplitude for the case of two different quark pairs, since the identical case can be obtained from it. The sole independent helicity amplitude can be labelled in terms of the helicities of the 1st quark, the 1st antiquark, the 2nd quark and the 2nd antiquark (in that order):

$$
\mathcal{M}^{+-+-}=i A g^{2} T_{i j}^{a} T_{k l}^{a}\left(\frac{\langle 24\rangle^{2}}{\langle 12\rangle\langle 34\rangle}+\frac{[13]^{2}}{[12][34]}\right) .
$$

The other helicity amplitudes can be obtained by parity and charge conjugation transformations:

$$
\begin{array}{ll}
\left|\mathcal{M}^{-+-+}\right|^{2} & \stackrel{P}{=}\left|\mathcal{M}^{+-+-}\right|^{2}, \quad\left|\mathcal{M}^{-++-}\right|^{2} \stackrel{C}{=}\left|\mathcal{M}^{+-+-}\right|_{1 \leftrightarrow 2}^{2}, \\
\left|\mathcal{M}^{+--+}\right|^{2} & \stackrel{C}{=}\left|\mathcal{M}^{+-+-}\right|_{3 \leftrightarrow 4}^{2} .
\end{array}
$$

\section{The Soft Higgs Limit}

The effective Lagrangian, Eq. 1, implies that for the case of constant Higgs field $H$, i.e., a Higgs boson with no momentum, the amplitude for a process containing a Higgs boson reduces to the amplitude for the process without the Higgs boson times an overall factor of $A$. For the $H g g g g$ amplitude only the helicity conserving ++-- amplitude survives in the $p_{\mathrm{H}} \rightarrow 0$ limit. In

this limit one can show that the two terms in Eq. 23 are equal, regardless of 
the ordering of the momenta. As an example, consider the subamplitude

$$
m\left(1^{-}, 3^{+}, 2^{-}, 4+\right)=-\frac{\langle 12\rangle^{4}}{\langle 13\rangle\langle 32\rangle\langle 24\rangle\langle 41\rangle}-\frac{[34]^{4}}{[13][32][24][41]} .
$$

In the limit $p_{\mathrm{H}} \rightarrow 0$ the second term in this expression becomes

$$
\begin{aligned}
\frac{[34]^{4}\langle 31\rangle\langle 23\rangle\langle 42\rangle\langle 14\rangle}{S_{13} S_{32} S_{24} S_{41}} & =\frac{\left\langle 3+\left|p_{4}\right| 2+\right\rangle\left\langle 3+\left|\not p_{4}\right| 1+\right\rangle\left\langle 4+\left|p_{3}\right| 1+\right\rangle\left\langle 4+\left|p_{3}\right| 2+\right\rangle}{S_{13} S_{32} S_{24} S_{41}} \\
& =\frac{\left\langle 3+\left|p_{1}\right| 2+\right\rangle\left\langle 3+\left|p_{2}\right| 1+\right\rangle\left\langle 4+\left|p_{2}\right| 1+\right\rangle\left\langle 4+\left|p_{1}\right| 2+\right\rangle}{S_{13} S_{32} S_{24} S_{41}} \\
& =\frac{\langle 12\rangle^{4}[13][32][24][41]}{S_{13} S_{32} S_{24} S_{41}}
\end{aligned}
$$

where momentum conservation as used in the second line. When common factors are cancelled Eq. 32 is identical to the first term of Eq. 31. The final result agrees with the well-known form of the pure gluon subamplitudes[11, 12, 13.

The $H q \bar{q} g g$ subamplitudes reduce to the $q \bar{q} g g$ subamplitudes:

$$
\begin{array}{ccc}
m^{+-+-}(3,4) & \stackrel{p_{\mathrm{H} \rightarrow 0}}{\rightarrow} & -\frac{2[13]^{3}}{[12][14][34]} \\
m^{+-+-}(4,3) & \stackrel{p_{\mathrm{H} \rightarrow 0}^{\rightarrow}}{\rightarrow} & -\frac{2\langle 14\rangle\langle 24\rangle^{2}}{\langle 12\rangle\langle 13\rangle\langle 34\rangle}
\end{array}
$$

Finally, the soft-Higgs limit of the $H q \bar{q} q^{\prime} \bar{q}^{\prime}$ amplitude is

$$
\mathcal{M}^{+-+-} \stackrel{p_{\mathrm{H}} \rightarrow 0}{\rightarrow}-2 i A g^{2} T_{i j}^{a} T_{k l}^{a} \frac{\langle 24\rangle^{2}}{\langle 12\rangle\langle 34\rangle},
$$

which has the proper relation to the amplitude for $q \bar{q} q^{\prime} \bar{q}^{\prime}$.

\section{Factorization of the Amplitudes}

The helicity amplitudes we have calculated factorize in the limit that a gluon becomes soft or two particles become collinear. This property has been established for the pure QCD processes involving quarks and gluons. We will present some representative examples of the factorization limits of our amplitudes. 
The simplest cases involve the reduction of the Higgs plus 3 particle amplitudes to the Higgs plus two gluon amplitude in the appropriate limit. Discussion of these limits is facilitated by expressing the $\mathrm{Hgg}, \mathrm{Hg} g \mathrm{~g}$, and $H q \bar{q} g$ amplitudes in the same dual color decompositions as we used for the $H g g g g$ and $H q \bar{q} g g$ amplitudes. For the $H g g$ case there is only one subamplitude, $m_{g g}(1,2)$, which can be obtained from Eq. 12 by replacing $\delta_{a b}$ by 1. For the Hggg case there are two subamplitudes but the Ward identity ensures that they are equal and opposite. The subamplitude $m_{g g g}(1,2,3)$ can be obtained from Eq. 14 by replacing $f_{a b c}$ by $-i$. The lone subamplitude for $H q \bar{q} g$ is Eq. 16 with the factor of $T_{i j}^{a}$ removed.

Taking one of the gluons to be soft in $m_{g g g}\left(1^{+}, 2^{+}, 3^{+}\right)$yields

$$
m_{g g g}\left(1^{+}, 2^{+}, 3^{+}\right) \stackrel{p_{1} \rightarrow 0}{\rightarrow}-\left\{\frac{g \sqrt{2}\langle 32\rangle}{\langle 12\rangle\langle 31\rangle}\right\} m_{g g}\left(2^{+}, 3^{+}\right)
$$

where the factor in brackets is the square root of the "eikonal factor". This is in keeping (up to phase conventions) with the general result of Mangano et al. [11]. A similar limit applies to $m_{g g g}\left(1^{-}, 2^{+}, 3^{+}\right)$. Taking two of the gluons to be collinear is accomplished by letting $p_{1} \rightarrow z P$ and $p_{2} \rightarrow(1-z) P$ :

$$
\begin{aligned}
& m_{g g g}\left(1^{+}, 2^{+}, 3^{+}\right) \rightarrow\left(\frac{i g \sqrt{2}[12]}{\sqrt{z(1-z)}}\right)\left(\frac{-i}{S_{12}}\right) m_{g g}\left(P^{+}, 3^{+}\right), \\
& m_{g g g}\left(1^{-}, 2^{+}, 3^{+}\right) \rightarrow\left(\frac{i g \sqrt{2}\langle 12\rangle(1-z)^{2}}{\sqrt{z(1-z)}}\right)\left(\frac{-i}{S_{12}}\right) m_{g g}\left(P^{+}, 3^{+}\right),
\end{aligned}
$$

which is again consistent with the general result 11 .

The Hgggg subamplitudes exhibit the same factorization properties. Notice that only the momenta which are adjacent in the argument of the subamplitude appear paired in the denominators. For example, taking $p_{1}$ to be soft in $m\left(1^{+}, 2^{+}, 3^{+}, 4^{+}\right)$gives

$$
m\left(1^{+}, 2^{+}, 3^{+}, 4^{+}\right) \stackrel{p_{1} \rightarrow 0}{\rightarrow} \frac{S_{234}^{2}}{\langle 12\rangle\langle 23\rangle\langle 34\rangle\langle 41\rangle}=\frac{\sqrt{2}\langle 42\rangle}{\langle 12\rangle\langle 41\rangle} m_{g g g}\left(2^{+}, 3^{+}, 4^{+}\right),
$$

in agreement with the general result. The $\mathcal{O}\left(1 / S_{i j}\right)$ singularities in Eq. 22 are in reality only $\mathcal{O}\left(1 / \sqrt{S_{i j}}\right)$ singularites. For example, taking $p_{1} \rightarrow z P$ and 


$$
\begin{aligned}
p_{2} & \rightarrow(1-z) P \text { in } m\left(1^{-}, 2^{+}, 3^{+}, 4^{+}\right) \text {gives } \\
& m\left(1^{-}, 2^{+}, 3^{+}, 4^{+}\right) \stackrel{1 \| 2}{\rightarrow} \frac{1}{\sqrt{z(1-z)}}\left\{\frac{(1-z)^{2}}{[12]} \frac{S_{P 34}^{2}}{\langle P 3\rangle\langle 34\rangle\langle 4 P\rangle}-\frac{z^{2}}{\langle 12\rangle} \frac{[34]^{3}}{[P 3][P 4]}\right\} \\
& =\frac{\sqrt{2}}{\sqrt{z(1-z)}}\left\{\frac{(1-z)^{2}}{[12]} m_{g g g}\left(P^{+}, 3^{+}, 4^{+}\right)-\frac{z^{2}}{\langle 12\rangle} m_{g g g}\left(P^{-}, 3^{+}, 4^{+}\right)\right\} .
\end{aligned}
$$

The $H q \bar{q} g g$ and $H q \bar{q} q^{\prime} \bar{q}^{\prime}$ amplitudes also factor. Taking the limit of $p_{1} \| p_{2}$ as before gives:

$$
\begin{aligned}
m_{q \bar{q} g g}^{+-+} & \stackrel{1 \| 2}{\rightarrow} \frac{(1-z)}{[12]} \frac{S_{P 34}}{\langle P 3\rangle\langle 34\rangle\langle 4 P\rangle}+\frac{z}{\langle 12\rangle} \frac{[34]^{3}}{[P 3][P 4]} \\
& =\frac{(1-z)}{[12]} m_{g g g}\left(P^{+}, 3^{+}, 4^{+}\right)+\frac{z}{\langle 12\rangle} m_{g g g}\left(P^{-}, 3^{+}, 4^{+}\right)
\end{aligned}
$$

and

$$
M_{q \bar{q} q^{\prime} \bar{q}^{\prime}}^{+-+} \stackrel{1 \| 2}{\longrightarrow}-i g^{2} T_{a b}^{a} T_{d c}^{a}\left\{\frac{(1-z)}{[12]} m_{q \bar{q} g}^{+-+}+\frac{z}{\langle 12\rangle} m_{q \bar{q} g}^{+--}\right\} .
$$

The Hgggg subamplitudes also factor in the three-gluon channel. Letting $P=p_{1}+p_{2}+p_{3}$ and taking $P^{2} \rightarrow 0$ in the -+++ subamplitude gives

$m\left(1^{-}, 2^{+}, 3^{+}, 4^{+}\right) \rightarrow[P 4]^{2} \frac{1}{P^{2}} \frac{\langle 1 P\rangle^{2}[23]^{2}}{S_{12} S_{23}} \sim m_{g g}(4+, P+) \frac{1}{P^{2}} \tilde{m}\left(1^{-}, 2^{+}, 3^{+}, P^{-}\right)$,

where $\tilde{m}$ is the four gluon subamplitude (without a Higgs boson) and the $\sim$ indicates equality modulo phases. Identical relations hold for the $1 / S_{124}$ and $1 / S_{134}$ poles. Since the four-gluon amplitude is helicity conserving the other helicity amplitudes have no three-gluon poles. Likewise there is no $1 / S_{234}$ pole in $m\left(1^{-}, 2^{+}, 3^{+}, 4^{+}\right)$.

\section{Numerical Results and Conclusions}

We will present numerical results for the CERN Large Hadron Collider (LHC) at a center-of-mass energy of $\sqrt{S}=14 \mathrm{TeV}$ and the Fermilab Tevatron at $\sqrt{S}=2 \mathrm{TeV}$. Since all the parton level cross sections are singular in the small 
$p_{\mathrm{T}}$ limit of one of the jets we will place a $p_{\mathrm{T}}$ cut on the outgoing jets. Since there are also collinear singularities we will require that the outgoing jets be separated by $\Delta R_{i j} \equiv \sqrt{\Delta \phi_{i j}^{2}+\Delta \eta_{i j}} \geq 0.7$. We will also require the outgoing jets have rapidity $|y|<2.5$. Since there are no singularities depending on the momentum of the Higgs boson we will allow it to be unconstrained, except for a $p_{\mathrm{T}}$ cut.

The results separated according to parton processes are presented in Fig.'s 7 and 8. We see that at the LHC the all gluon process dominates as expected with the $q g \rightarrow q g H$ process and its charge conjugate contributing an additional 15\%. The other processes are negligible. At the Tevatron the pure gluon process and the $q g \rightarrow q g H$ process give roughly equal contributions.

Fig.'s 9 and 10 show the result of varying the transverse momentum cut (on the jets and the Higgs boson simultaneously). We see that in both cases the cross section drops sharply with the increasing $p_{\mathrm{T}}$ cut. The dependence of the cross section on the other cuts is weak. Increasing the minimum $\Delta R$ to 1.0 decreases the cross section by about $15 \%$. Requiring that the jets be separated from the Higgs boson the same $\Delta R$ also reduces the cross section be about $15 \%$.

In summary, we have presented the amplitudes for the production of a Higgs accompanied by two jets. We find that the cross section is around a few picobarns at the LHC and a few hundredths of a pb at the Tevatron. Our results provide the "real" corrections to Higgs production at non-zero transverse momentum. They can be combined with the virtual corrections to complete the next-to-leading order calculation. 


\section{Appendix. Squaring the Amplitudes}

We first proceed to verify the incoherence of the subamplitudes for $g g g g H$. Using the fact that subamplitudes are invariant (for $n=4$ ) under reversal of the order of the arguments we write

$$
\begin{aligned}
\mathcal{M} & =\left\{\left[\operatorname{tr}\left(T^{a} T^{b} T^{c} T^{d}\right)+\operatorname{tr}\left(T^{d} T^{c} T^{b} T^{a}\right)\right] m(1,2,3,4)\right. \\
& +\left[\operatorname{tr}\left(T^{a} T^{b} T^{d} T^{d}\right)+\operatorname{tr}\left(T^{d} T^{d} T^{b} T^{a}\right)\right] m(1,2,4,3) \\
& \left.+\left[\operatorname{tr}\left(T^{a} T^{c} T^{b} T^{d}\right)+\operatorname{tr}\left(T^{d} T^{b} T^{c} T^{a}\right)\right] m(1,3,2,4)\right\} .
\end{aligned}
$$

It is straightforward to show that the squared color factors are

$$
\begin{aligned}
c_{1} & =\sum_{\text {colors }}\left[\operatorname{tr}\left(T^{a} T^{b} T^{c} T^{d}\right)+\operatorname{tr}\left(T^{d} T^{c} T^{b} T^{a}\right)\right]^{2} \\
& =\frac{\left(N^{2}-1\right)^{2}}{4 N^{2}}+\frac{1}{16}\left[\left(\frac{5}{3}\right)^{2}\left(N^{2}-1\right)+N^{2}\left(N^{2}-1\right)\right]
\end{aligned}
$$

and that the cross terms are

$$
\begin{aligned}
c_{2} & =\sum_{\text {colors }}\left[\operatorname{tr}\left(T^{a} T^{b} T^{c} T^{d}\right)+\operatorname{tr}\left(T^{d} T^{c} T^{b} T^{a}\right)^{*}\right]\left[\operatorname{tr}\left(T^{a} T^{b} T^{d} T^{c}\right)+\operatorname{tr}\left(T^{c} T^{d} T^{b} T^{a}\right)^{*}\right] \\
& =\frac{\left(N^{2}-1\right)^{2}}{4 N^{2}}+\frac{1}{16}\left[\left(\frac{5}{3}\right)^{2}\left(N^{2}-1\right)-N^{2}\left(N^{2}-1\right)\right]
\end{aligned}
$$

The amplitude squared is then

$$
\begin{aligned}
\sum_{\text {colors }}|\mathcal{M}|^{2} & =4 g^{4} A^{2}\left\{c_{1}\left[|m(1,2,3,4)|^{2}+|m(1,2,4,3)|^{2}+|m(1,3,2,4)|^{2}\right]\right. \\
& +c_{2}\left[m(1,2,3,4)\left(m(1,2,4,3)^{*}+m(1,3,2,4)^{*}\right)\right. \\
& +m(1,2,4,3)\left(m(1,2,3,4)^{*}+m(1,3,2,4)^{*}\right) \\
& \left.\left.+m(1,3,2,4)\left(m(1,2,3,4)^{*}+m(1,2,4,3)^{*}\right)\right]\right\}
\end{aligned}
$$

Use of the Ward identity, Eq. (19), allows all the cross terms to be written as subamplitudes squared. When this is done and the above results for the color factor are used, Eq. (20) follows.

The following identities were used to relate the spinor products to traces over gamma matrices [11]:

$$
\begin{aligned}
{\left[i_{1} i_{2}\right]\left\langle i_{2} i_{3}\right\rangle \ldots\left\langle i_{2 n} i_{1}\right\rangle } & =\left\{i_{1} i_{2} \ldots i_{2 n} P_{+}\right\} \\
\left\langle i_{1} i_{2}\right\rangle\left[i_{2} i_{3}\right] \ldots\left[i_{2 n} i_{1}\right] & =\left\{i_{1} i_{2} \ldots i_{2 n} P_{-}\right\}
\end{aligned}
$$


where $P_{ \pm}=\frac{1}{2}\left(1 \pm \gamma_{5}\right)$, and $\left\{i_{1} i_{2} \ldots i_{n}\right\}$ denotes the trace of $\not p_{1} \not_{2} \ldots p_{n}$. In order to reduce the traces which contained a factor of $\gamma_{5}$, we use the identity[11]:

$$
\begin{aligned}
\left\{i_{1} i_{2} \ldots i_{2 n} \gamma_{5}\right\} & \left\{j_{1} j_{2} \ldots j_{2 m} \gamma_{5}\right\}=\left\{i_{1} i_{2} \ldots i_{2 n}\right\}\left\{j_{1} j_{2} \ldots j_{2 m}\right\} \\
& -2\left[\left[i_{1} i_{2}\right]\left\langle i_{2} i_{3}\right\rangle \ldots\left\langle i_{2 n} i_{1}\right\rangle\left\langle j_{1} j_{2}\right\rangle\left[j_{2} j_{3}\right] \ldots\left[j_{2 n} j_{1}\right]+c . c .\right]
\end{aligned}
$$

We first consider the square of the $g g g g H$ subamplitudes. For the case of $m\left(1^{+}, 2^{+}, 3^{+}, 4^{+}\right)$, we have

$$
\left|m\left(1^{+}, 2^{+}, 3^{+}, 4^{+}\right)\right|^{2}=\frac{M_{\mathrm{H}}^{8}}{S_{12} S_{23} S_{34} S_{41}} .
$$

In the case of $m\left(1^{-}, 2^{+}, 3^{+}, 4^{+}\right)$, we rewrite Eq. 22 in a more compact form:

$$
\begin{aligned}
& m\left(1^{-}, 2^{+}, 3^{+}, 4^{+}\right)=-\frac{\left\langle 1-\left|p_{\mathrm{H}}\right| 3-\right\rangle^{2}[24]^{2}}{S_{124} S_{12} S_{14}}-\frac{\left\langle 1-\left|p_{\mathrm{H}}\right| 4-\right\rangle^{2}[23]^{2}}{S_{123} S_{12} S_{23}} \\
& -\frac{\left\langle 1-\left|p_{\mathrm{H}}\right| 2-\right\rangle^{2}[34]^{2}}{S_{134} S_{14} S_{34}}+\frac{[24]}{[12][14]\langle 13\rangle}\left\{\frac{\left\langle 1-\left|p_{\mathrm{H}}\right| 2-\right\rangle^{2}}{\langle 14\rangle\langle 34\rangle}+\frac{\left\langle 1-\left|p_{\mathrm{H}}\right| 4-\right\rangle^{2}}{\langle 12\rangle\langle 23\rangle}\right\} .
\end{aligned}
$$

We then write

$$
\left|m\left(1^{-}, 2^{+}, 3^{+}, 4^{+}\right)\right|^{2}=\sum_{i=1}^{5} \sum_{j=1}^{i} \frac{n_{i j}}{d_{i} d_{j}},
$$

where the independent terms are:

$$
\begin{aligned}
n_{11}= & \frac{1}{4} S_{24}^{2}\{1(2+4) 3(2+4)\}^{2}, \quad n_{44}=\frac{1}{4} S_{12} S_{13} S_{24} S_{34}\{1(3+4) 2(3+4)\}^{2} \\
n_{12}= & \{1(2+4) 324(2+3)\}^{2}-2 S_{23} S_{24}\left(S_{12} S_{24}+S_{13} S_{34}+\{1243\}\right) \\
n_{14}= & -S_{24}[\{1(2+4) 342(3+4)\}\{1(2+4) 312(3+4)\} \\
-4 n_{24}= & \quad-\{1(2+3) 432(3+4)\}\{1(2+3) 42\}\{132(3+4)\} \\
& -\{1(2+3) 432(3+4)\}\left(\{1234\}^{2}-4 S_{12} S_{23} S_{34} S_{41}\right) \\
& -[\{1(2+3) 432(3+4)\}\{1234\} \\
& \left.+2 S_{23} S_{34}\left(S_{12}\{1324\}-S_{13}\{1234\}+S_{14}\{1243\}+2 S_{12} S_{14} S_{24}\right)\right] \\
& \times(\{1(2+3) 42\}-\{132(3+4)\})
\end{aligned}
$$




$$
\begin{aligned}
n_{25}= & -\frac{1}{4} S_{23}\{1(2+3) 4(2+3)\}^{2}\{1324\} \\
n_{45}= & -S_{13} S_{24}[\{1(3+4) 234(2+3)\}\{1(3+4) 214(2+3)\} \\
& \left.\quad-\{1234\}\left(S_{13} S_{23}+S_{14} S_{24}+\{1324\}\right)\left(S_{12} S_{24}+S_{13} S_{34}+\{1243\}\right)\right] \\
d_{1}= & S_{12} S_{14} S_{124}, \quad d_{4}=S_{12} S_{13} S_{14} S_{34} .
\end{aligned}
$$

The remaining terms can be obtained by switching the momenta:

$$
\begin{aligned}
& n_{22}=n_{11}(3 \leftrightarrow 4), \quad n_{33}=n_{11}(2 \leftrightarrow 3), \quad n_{55}=n_{44}(2 \leftrightarrow 4), \\
& n_{13}=n_{12}(2 \leftrightarrow 4), \quad n_{15}=n_{14}(2 \leftrightarrow 4), \quad n_{23}=n_{13}(3 \leftrightarrow 4), \\
& n_{34}=n_{25}(2 \leftrightarrow 4), \quad n_{35}=n_{24}(2 \leftrightarrow 4), \quad d_{5}=d_{4}(2 \leftrightarrow 4), \\
& d_{2}=d_{1}(1 \leftrightarrow 2,3 \leftrightarrow 4), \quad d_{3}=d_{1}(1 \leftrightarrow 4,2 \leftrightarrow 3) .
\end{aligned}
$$

The two independent permutations of $m\left(1^{-}, 2^{-}, 3^{+}, 4^{+}\right)$squared are:

$$
\begin{aligned}
\left|m\left(1^{-}, 2^{-}, 3^{+}, 4^{+}\right)\right|^{2}= & \frac{S_{12}^{3}}{S_{14} S_{23} S_{34}}+\frac{S_{34}^{3}}{S_{12} S_{14} S_{23}}+\frac{\{1234\}^{2}-2 S_{12} S_{23} S_{34} S_{41}}{S_{14}^{2} S_{23}^{2}} \\
\left|m\left(1^{-}, 3^{+}, 2^{-}, 4^{+}\right)\right|^{2}= & \frac{S_{12}^{4}+S_{34}^{4}}{S_{13} S_{14} S_{23} S_{34}} \\
& +\left[\left(\{1234\}^{2}-2 S_{12} S_{23} S_{34} S_{41}\right)\left(\{1243\}^{2}-2 S_{12} S_{24} S_{43} S_{31}\right)\right. \\
& \left.+\{1234\}\{1243\}\left(\{1234\}\{1243\}+2 S_{12} S_{34}\{1324\}\right)\right] \\
& /\left[2\left(S_{13} S_{14} S_{23} S_{34}\right)^{2}\right] .
\end{aligned}
$$

Turning our attention to the $q \bar{q} g g H$ amplitudes, we break the subamplitude $m^{+-++}(3,4)$ into a symmetric piece, $m_{s}$, and anti-symmetric piece, $m_{a}$, under interchange of the two gluons. The squared amplitude is then given by

$$
\left|\mathcal{M}^{+-++}\right|^{2}=2 A^{2} g^{4}\left[C_{1}\left(\left|m_{s}\right|^{2}+\left|m_{a}\right|^{2}\right)+C_{2}\left(\left|m_{s}\right|^{2}-\left|m_{a}\right|^{2}\right)\right],
$$

where the color factors are $C_{1}=\frac{\left(N^{2}-1\right)^{2}}{4 N}$ and $C_{2}=-\frac{\left(N^{2}-1\right)}{4 N}$. We write:

$$
\begin{aligned}
& \left|m_{s}\right|^{2}=\frac{1}{4}\left(\frac{n_{11}}{d_{1}^{2}}+\frac{n_{22}}{d_{2}^{2}}+\frac{n_{12}}{d_{1} d_{2}}\right) \\
& \left|m_{a}\right|^{2}=\frac{n_{11}}{d_{3}^{2}}+\frac{n_{22}}{d_{4}^{2}}+\frac{n_{33}}{d_{5}^{2}}-\frac{n_{12}}{d_{3} d_{4}}+\frac{n_{13}}{d_{3} d_{5}}+\frac{n_{23}}{d_{4} d_{5}}
\end{aligned}
$$


where the numerator and denominator terms are

$$
\begin{aligned}
n_{11}= & \frac{1}{4} S_{14} S_{24}\{2(1+4) 3(1+4)\}^{2}, \quad n_{22}=n_{11}(3 \leftrightarrow 4), \\
n_{33}= & \frac{1}{4} S_{12} S_{23} S_{24} S_{34}\{1(3+4) 2(3+4)\}^{2}, \\
n_{12}= & \{2(1+4) 314(1+3)\}\{2(1+4) 324(1+3)\} \\
& -\{1324\}\left(S_{12} S_{13}+S_{24} S_{34}+\{1243\}\right)\left(S_{12} S_{14}+S_{23} S_{34}+\{1234\}\right) \\
n_{13}= & -S_{24} n_{12}(2 \leftrightarrow 4), \quad n_{23}=-S_{23} n_{13}(3 \leftrightarrow 4), \\
d_{1}= & S_{14} S_{24} S_{124}, \quad d_{2}=S_{13} S_{23} S_{123}, \quad d_{5}=S_{12} S_{23} S_{24} S_{34}, \\
d_{4}= & S_{23} S_{123}\left(\frac{1}{S_{12}}+\frac{1}{2 S_{13}}\right)^{-1}, \quad d_{3}=S_{24} S_{124}\left(\frac{1}{S_{12}}+\frac{1}{2 S_{14}}\right)^{-1} .
\end{aligned}
$$

For the other independent helicity amplitude, squaring yields

$$
\begin{aligned}
\left|\mathcal{M}^{+-+-}\right|^{2}=g^{4} A^{2}\left\{C_{1}\left(\left|m^{+-+-}(3,4)\right|^{2}+\left|m^{+-+-}(4,3)\right|^{2}\right)\right. \\
\left.+2 C_{2} \operatorname{Re}\left[m^{+-+-}(3,4) m^{+-+-}(4,3)^{*}\right]\right\},
\end{aligned}
$$

where

$$
\begin{aligned}
& \left|m^{+-+-}(3,4)\right|^{2}=\left\{\frac{S_{13}^{3}}{S_{12} S_{14} S_{34}}+\frac{S_{24}^{3}}{S_{12} S_{23} S_{34}}+\frac{1}{S_{14} S_{23} S_{12}^{2} S_{34}^{2}}\right. \\
& \left.\times\left[-\{1243\}^{2}\{1324\}-S_{13} S_{24}\{1234\}\{1243\}+S_{12} S_{13} S_{24} S_{34}\{1324\}\right]\right\} \\
& \left|m^{+-+-}(4,3)\right|^{2}=\left\{\frac{S_{13}^{2} S_{23}}{S_{12} S_{24} S_{34}}+\frac{S_{14} S_{24}^{2}}{S_{12} S_{13} S_{34}}+\frac{\left(\{1243\}\{1234\}+S_{12} S_{34}\{1324\}\right)}{S_{12}^{2} S_{34}^{2}}\right\} \\
& 2 \operatorname{Re}\left[m^{+-+-}(3,4) m^{+-+-}(4,3)^{*}\right]=-\frac{\{1324\}}{S_{12} S_{34}}\left(\frac{S_{13}^{2}}{S_{14} S_{24}}+\frac{S_{24}^{2}}{S_{13} S_{23}}\right)
\end{aligned}
$$

For the $q \bar{q} q^{\prime} \bar{q}^{\prime}$ amplitude, the square of Eq. 29 yields

$$
\mathcal{M}^{2}=\frac{A^{2} g^{4}\left(N^{2}-1\right)}{4 S_{12} S_{34}}\left[\left(S_{13}-S_{24}\right)^{2}+\frac{\{1243\}^{2}}{S_{12} S_{34}}\right] .
$$

In the case of identical quark pairs, there is a second diagram whose square can be obtained by switching $1 \leftrightarrow 3$ in Eq. 61. The interference term which 
arises is

$-2 \operatorname{Re}\left[\mathcal{M M}^{*}(1 \leftrightarrow 3)\right]=\frac{-A^{2} g^{4}\left(N^{2}-1\right)}{4 N}\left[\frac{\left(S_{13}-S_{24}\right)^{2}\{1234\}-2\{1324\}\{1243\}}{S_{12} S_{23} S_{14} S_{34}}\right]$.

\section{ACKNOWLEDGMENTS}

The author's would like to thank S. Dawson for her work on the initial stages of this project and for helpful discussions. S.D. and D.R. would like to thank the Hackman Scholar program at Franklin and Marshall College for financial support.

\section{References}

[1] D. Buskulic et al. (ALEPH Coll.), Phys. Lett. B313 (1993) 299; P. Abreu et al. (DELPHI Coll.), Nucl. Phys. B421 (1994) 2; O. Adriani et al. (L3 Coll.), Phys. Lett B303 (1993) 391; R. Akers et al. (OPAK Coll.), Phys. Lett B327 (1994) 397.

[2] S. Dawson and R.P. Kauffman, Phys. Rev. Lett. 68 (1992) 2273.

[3] F. Abe et al. (CDF Coll.), Phys. Rev. Lett. 74 (1995) 2626; S. Abachi et al. (Dø Coll.), Phys. Rev. Lett. 74 (1995) 2632.

[4] A. Vainshtein, M. Voloshin, V. Zakharov, and M. Shifman, Sov. J. Nucl. Phys. 30 (1979) 429.

[5] S. Dawson, Nucl. Phys. B359 (1991) 283.

[6] A. Djouadi, M. Spira, and P. Zerwas, Phys. Lett. B264 (1991) 440.

[7] R.P. Kauffman, Phys. Rev. D 44, 1415 (1991).

[8] Z. Xu, D. Zhang, and L. Chang, Nucl. Phys. B291 (1987) 392.

[9] F. Berends et. al., Nucl. Phys. B206 (1982)61; ibid. B239 (1984) 382; ibid. B239 (1984) 395; ibid. B254 (1986) 265. 
[10] I. Hinchliffe and S.F. Novaes, Phys. Rev. D 38, 3475 (1988).

[11] M. Mangano, S. Parke, and Z. Xu, Nucl. Phys. B298 (1988) 653.

[12] M. Mangano and S. Parke, Nucl. Phys. B299 (1988) 673.

[13] M. Mangano and S. Parke, Phys. Reps. 200 (1991) 301.

[14] Z. Kunszt, Nucl. Phys. B271 (1986) 333. 


\section{FIGURE CAPTIONS}

Figure 1. The vertices and Feynman rules of the effective theory. The curly lines indicate gluons and the dashed lines indicate the Higgs boson.

Figure 2. The Feynman diagrams for the $g g g H$ amplitude. There are two more diagrams of the same form as b), where the Higgs boson attaches to gluon 1 and gluon 3 .

Figure 3. The Feynman diagram for the $q \bar{q} g H$ amplitude.

Figure 4. The Feynman diagrams for the $g g g g H$ amplitude. There are 12 diagrams of type a), 3 of type b), 1 of type c), 4 of type d), and 6 of type e), for a total of 26 .

Figure 5. The Feynman diagrams for the $q \bar{q} g g H$ amplitude. There is one diagram of type a), two of type b), 4 of type c) and one of type d), for a total of 8 .

Figure 6. The Feynman diagram for the $q \bar{q} q^{\prime} \bar{q}^{\prime} H$ amplitude. In the case when the quark pairs are identical there is a second diagram with the quark lines switched.

Figure 7. The various contributions to the cross section for production of a Higgs boson plus two jets at the LHC as a function of the mass of the Higgs boson. A $p_{\mathrm{T}}$ cut of $50 \mathrm{GeV}$ has been placed on the jets and the Higgs boson. The labels are as follows: $g g g g$ represents $g g \rightarrow g g H$; $q g q g$ represents $q g \rightarrow q g H$ and its complex conjugate; ggqq represents $g g \rightarrow q \bar{q} H ; q q q q$ represents all the processes involving two incoming quarks or antiquarks and two outgoing quarks or antiquarks; ggqq represents $g g \rightarrow q \bar{q} H$.

Figure 8. The various contributions to the cross section for production of a Higgs boson plus two jets at the Tevatron as a function of the mass of the Higgs boson. A $p_{\mathrm{T}}$ cut of $25 \mathrm{GeV}$ has been placed on the jets and the Higgs boson. The labels are the same as in Fig. 7.

Figure 9. The cross section for production of a Higgs boson plus two jets at the LHC for three values of the $p_{\mathrm{T}}$ cut.

Figure 10. The cross section for production of a Higgs boson plus two jets at the Tevatron for three values of the $p_{\mathrm{T}}$ cut. 


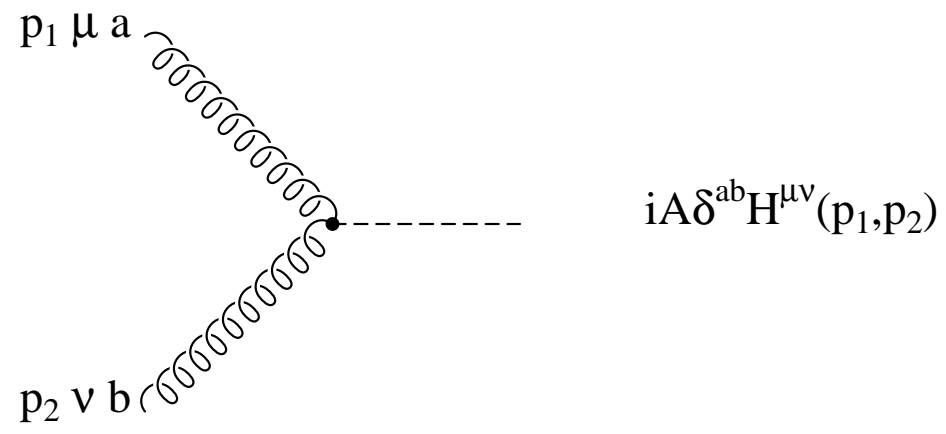

(a)
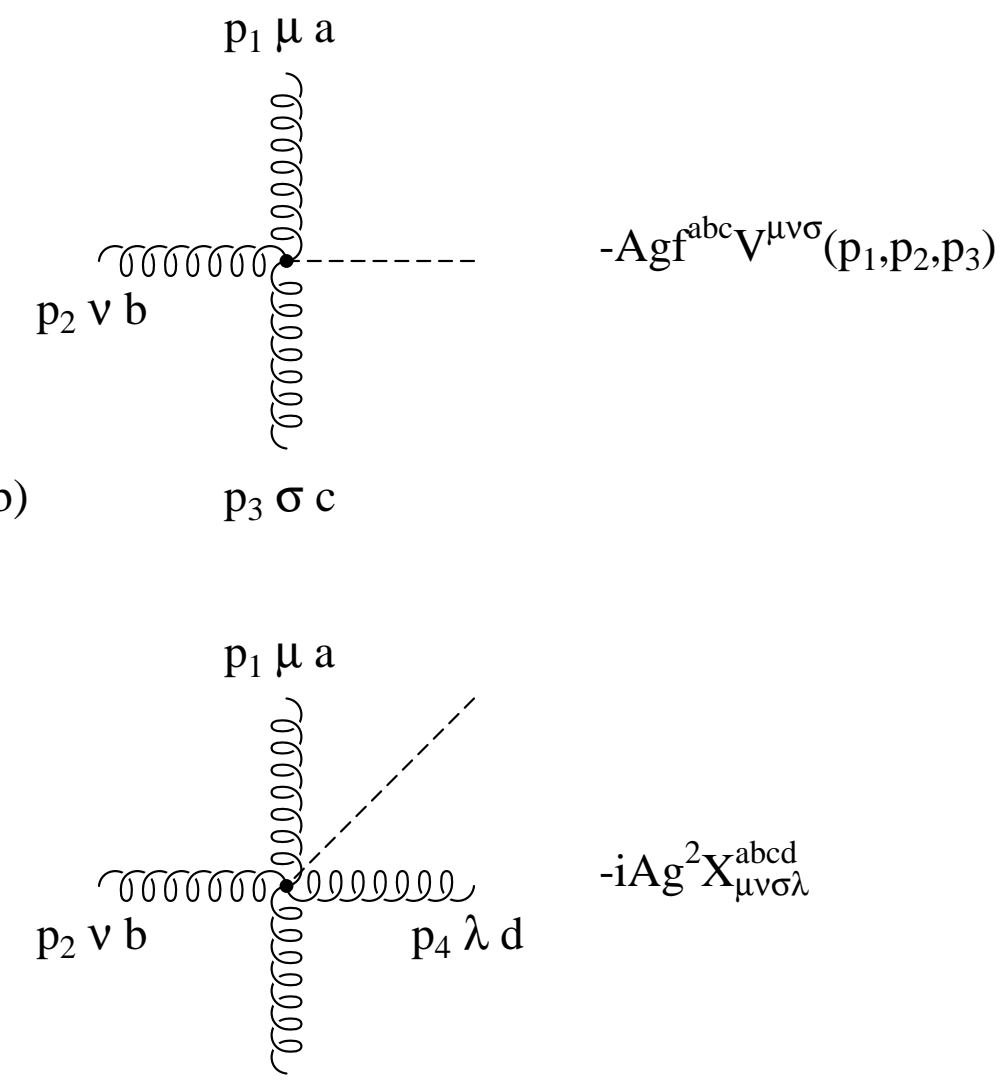

(c) $\quad \mathrm{p}_{3} \sigma \mathrm{c}$

Figure 1 


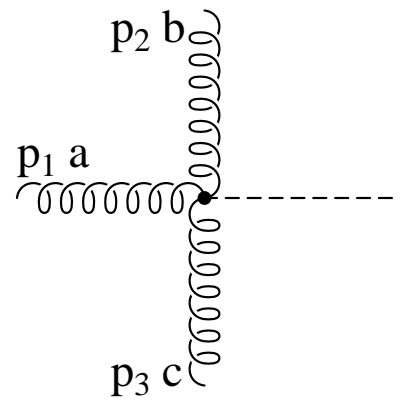

(a)

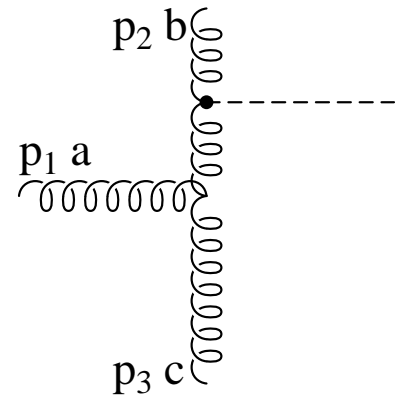

(b)

Figure 2 


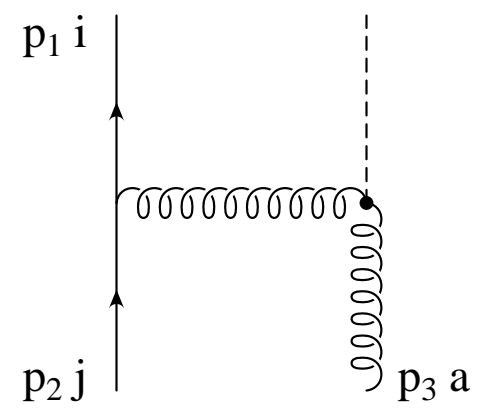

Figure 3 

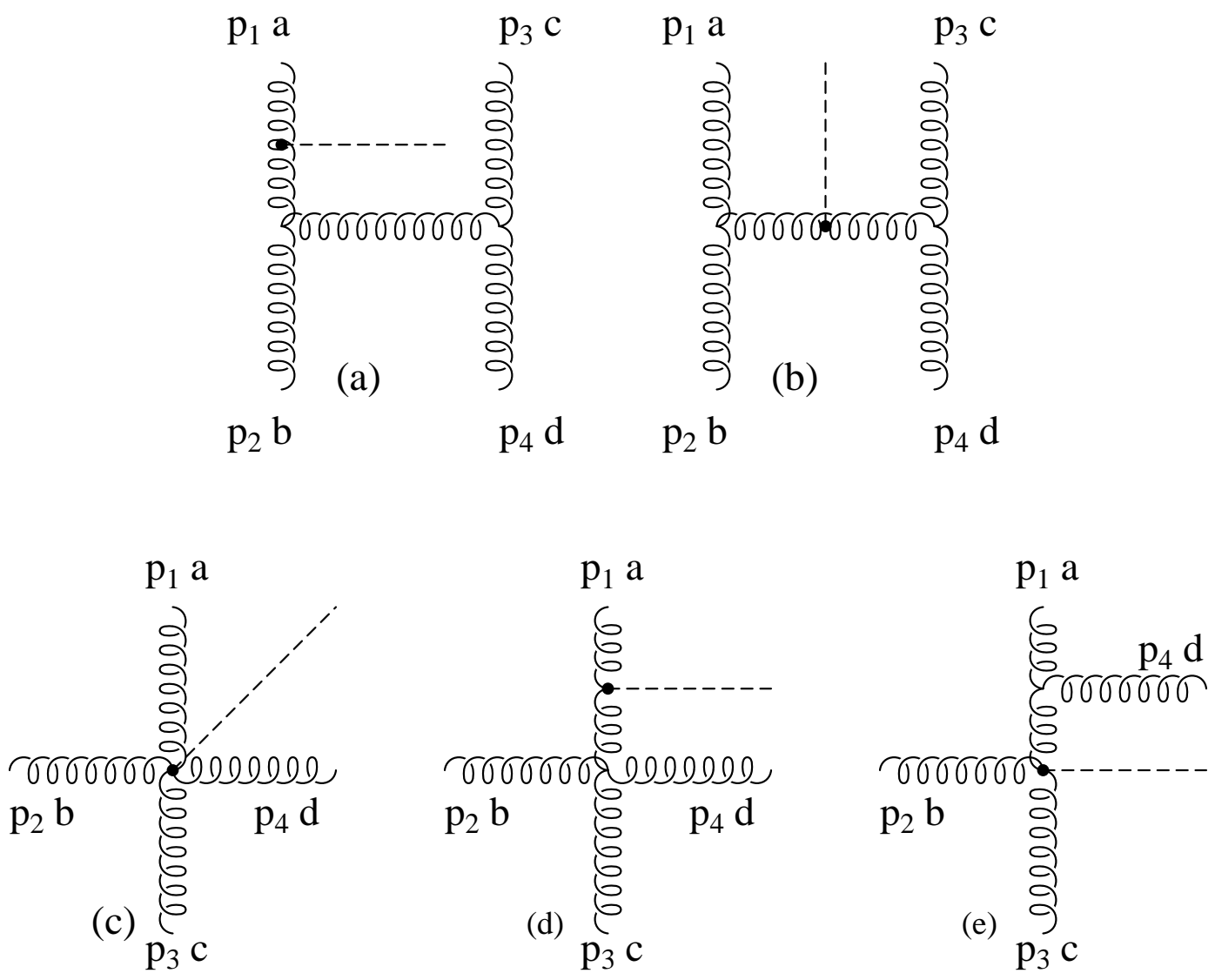

Figure 4 


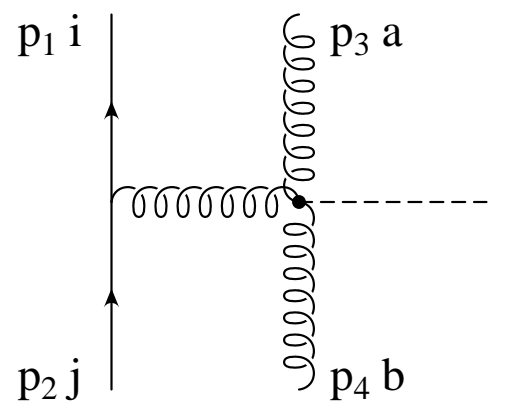

(a)

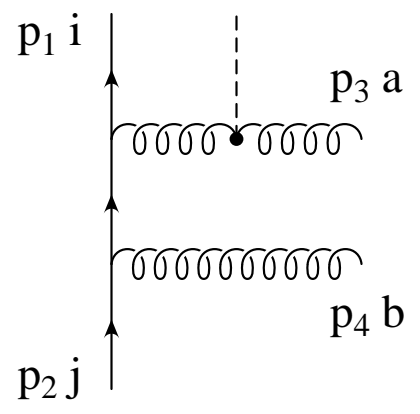

(c)

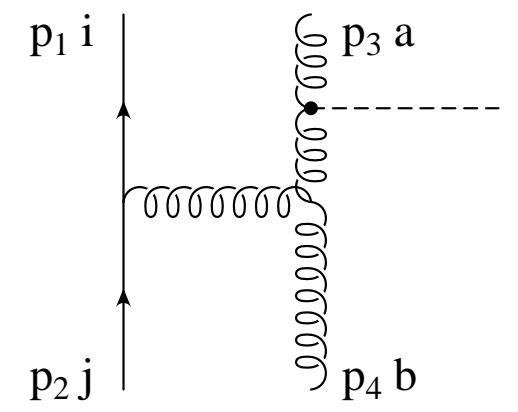

(b)

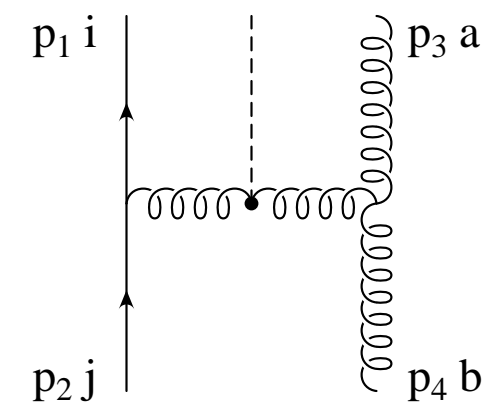

(d)

Figure 5 


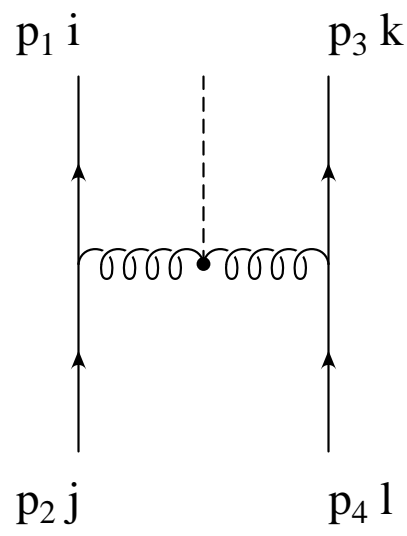

Figure 6 


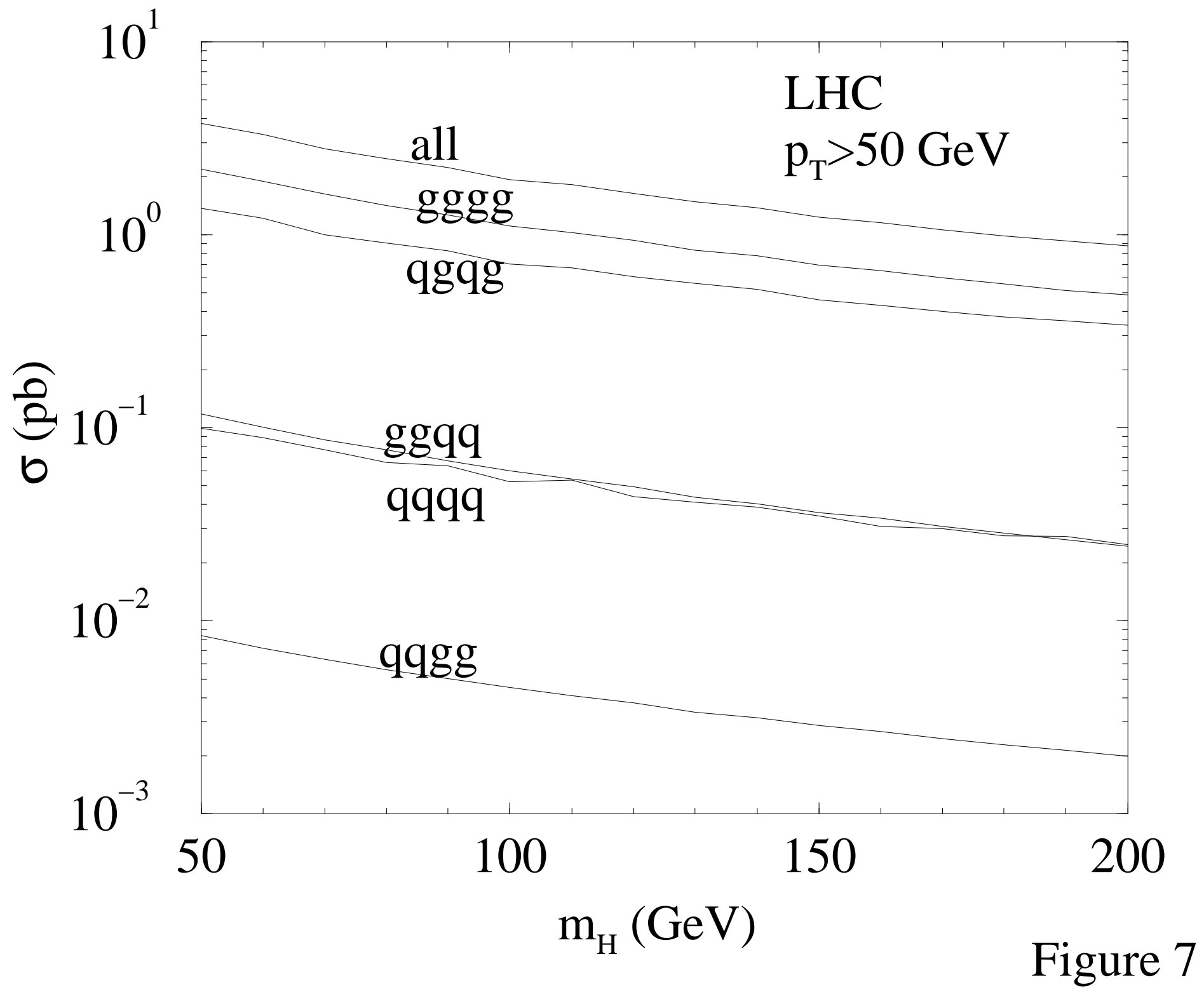




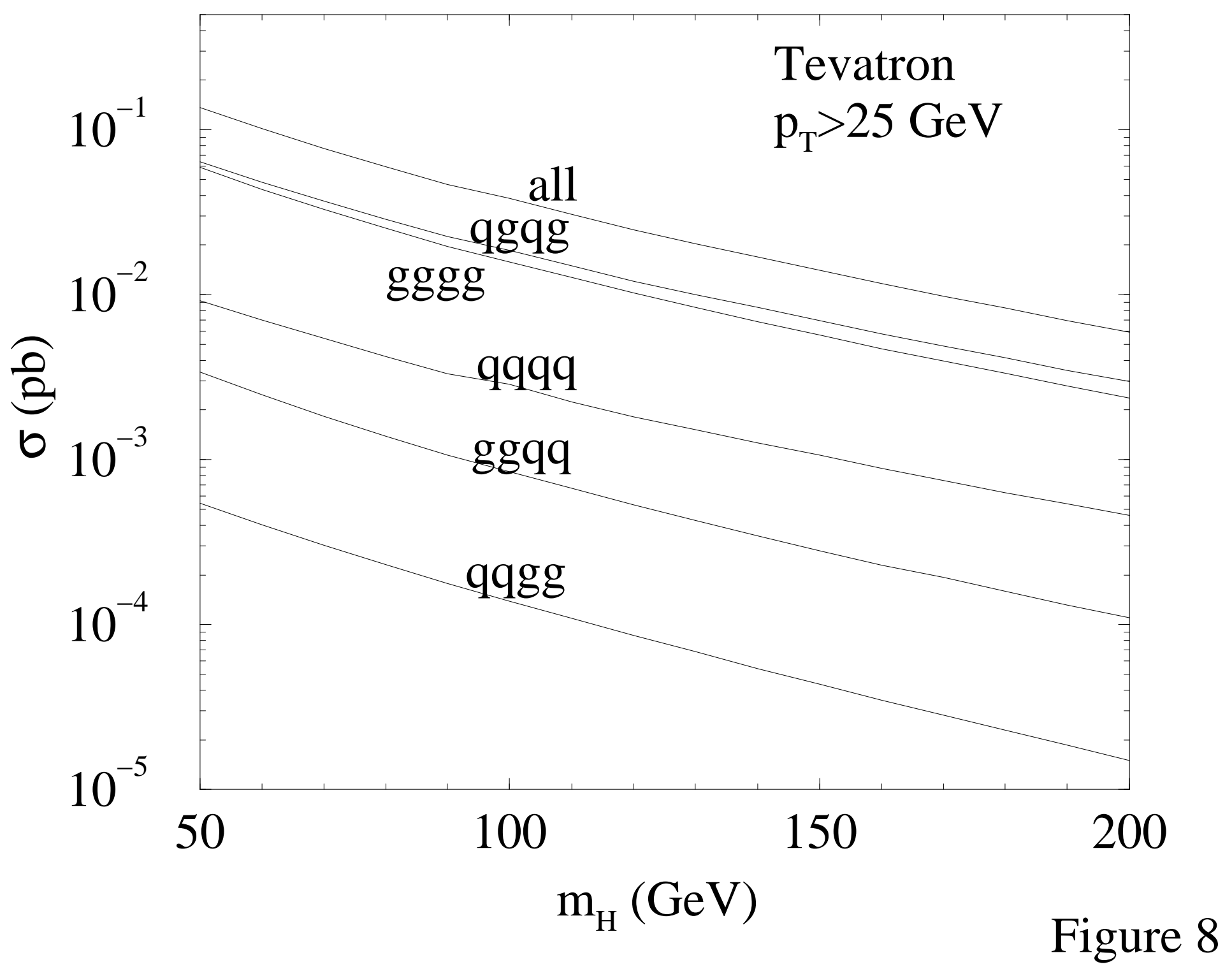




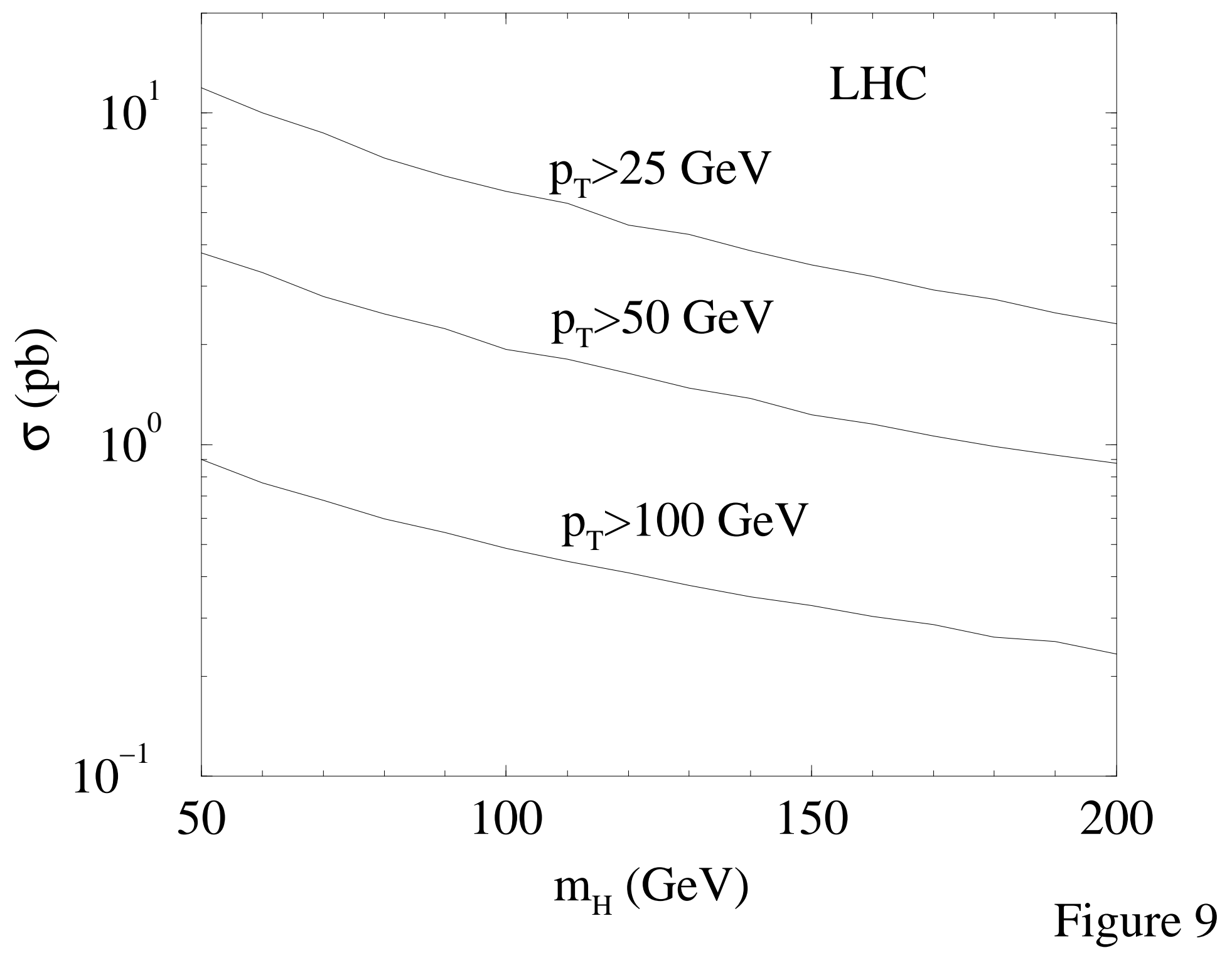




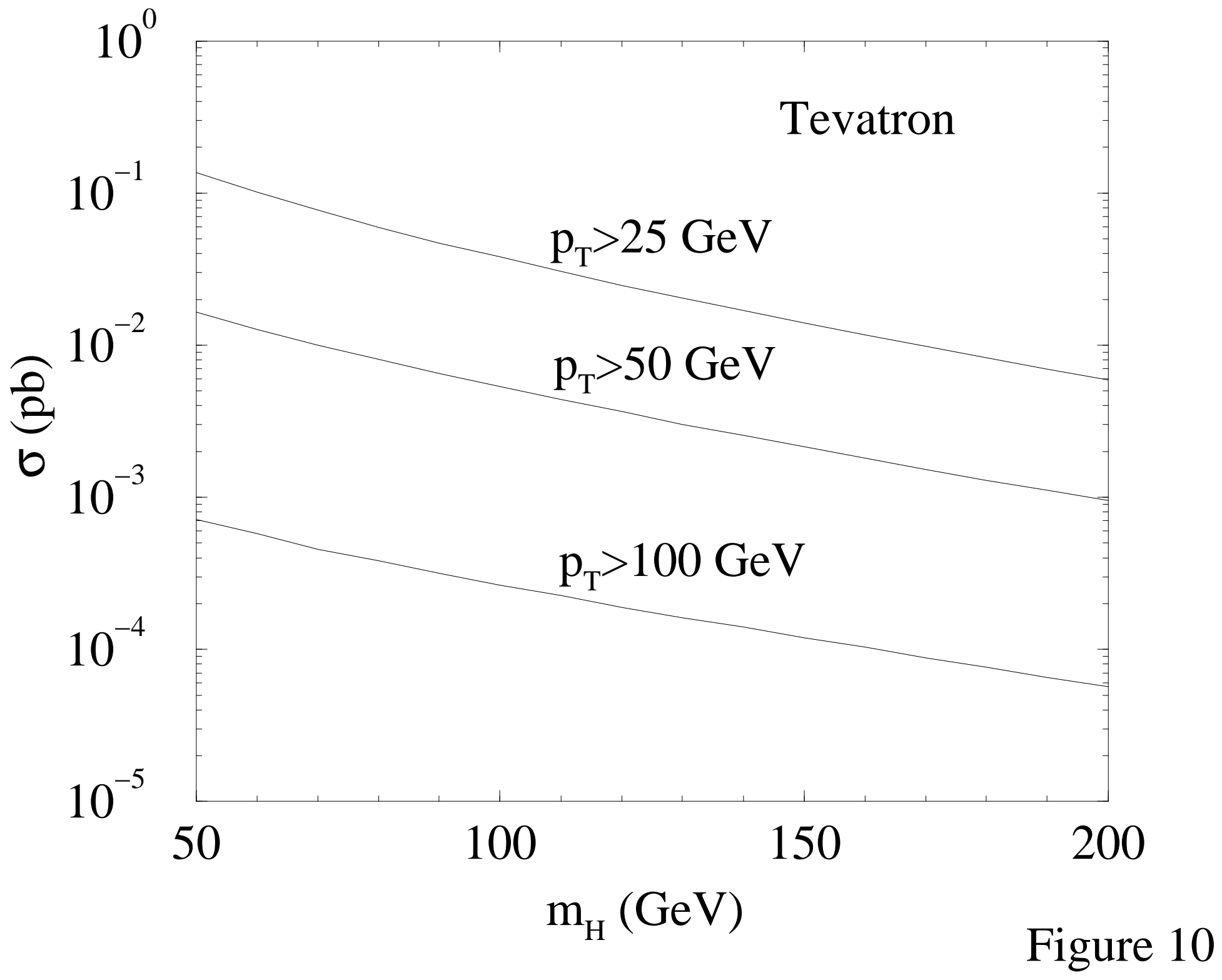

